\title{
Use of animals on expiring protocols
}

Emma Vale was new to the IACUC Office of Great Eastern University and had what she thought was a simple question: "After three years of research, when an IACUC protocol is submitted for full review as a renewing protocol, how should I handle any animals that are left on the expiring protocol?" Larry Covelli, the IACUC chairman, asked her to explain what she meant. "Well," she said, "should I transfer the remaining animals from the old protocol onto the new protocol, and if so, should I decrease the number of animals approved on the new protocol? I'm concerned because if I don't do anything, then the PI [Principal Investigator] will have animals remaining from the expiring protocol in addition to the animals that the IACUC has approved for the new protocol. Or should I tell the investigators to euthanize the animals that are left and then buy or breed more animals for the new protocol? Or is there something else I'm supposed to do?"

Covelli's first thought was to tell her to transfer the animals from the expiring protocol onto the new one and decrease the newly approved number, just like she would do if the animals had been purchased instead of transferred. Then he began to see the consequences. For example, if the IACUC approved 500 mice for new experiments, but the PI already had 50 in the animal facility, then the PI could order only 450 more mice for the new set of experiments. Simply transferring animals from an expiring protocol to a renewed one would not be useful if the transferred animals were of the wrong strain, genotype, age or sex. Also, as a scientist himself,
Covelli knew that PIs might have ongoing experiments to complete on the expiring protocol, but he wasn't sure whether the PIs included full descriptions of those ongoing studies on their newly submitted protocols. He suspected that they did not. So Covelli had a problem. Not only was there a need to address third-year renewing protocols that were funded through the Public Health Service, but he wasn't sure how to approach those protocols that required annual review under the US Department of Agriculture regulations. Covelli wasn't sure how the issue had been handled in the past, but he knew that the IACUC had to make it right in the future.

What would you suggest that Covelli and the Great Eastern IACUC should do to resolve this problem?

\section{RESPONSE}

\section{Transferring animals responsibly}

\author{
Tracy M. Heenan, DVM, CPIA \& \\ Michelle Keys, LATG, CPIA
}

Although they don't specifically address this issue, regulations such as the US Government Principles for the Utilization and Care of Vertebrate Animals Used in Testing, Research and Training ${ }^{1}$ and the Guide for the Care and Use of Laboratory Animals ${ }^{2}$ do stress the importance of using the minimum number of animals necessary to obtain valid experimental results. Any investigator who uses animals has a moral and regulatory imperative to be a good steward of animal resources and should plan studies accordingly to reduce unnecessary animal use.

A PI has various options for handling animals remaining on an expiring application. (i) Complete current studies on the expiring application so that there are no remaining animals to be transferred. (ii) Account for the remaining experimental procedures as well as the animal numbers necessary to complete those procedures in the 'continuation' application. (iii) Transfer animals of the correct strain, genotype, sex and age to his or her other approved applications. (iv) Transfer animals to other PIs' approved applications through a formalized 'animal transfer' process regulated by the institution. (v) Formally donate or transfer surplus animals for use in the institution's hands-on animal handling training courses. (vi) Euthanize existing animals that cannot be transferred or shared (the least desirable option).

The process of transferring animals from one application to another may be complicated, but problems can be minimized if the institution has established standard operating procedures and clearly defined expectations. By maintaining control of animal transfers through administrative, electronic and manual mechanisms to meet institutional needs and Public Health Service and USDA Policy requirements, the institution can ascertain that animals are transferred appropriately to approved applications. Procedures for transfer of animals should ensure that animals are properly accounted for on the respective protocols, that animals are transferred only to approved applications using that species and that multiple major survival surgical procedures are not done on individual animals without IACUC approval.

An email list-serve or group is a helpful way for PIs to alert other researchers of available animals. Sharing animals also contributes to the 'reduction' principle of the 3R's. Donation of animals, through a formalized process, to institutional animal handling training classes is an excellent way to use surplus animals and reduce unnecessary animal use.

The IACUC can alert a PI several months prior to an application's expiration of the need to either use the remaining animals for experiments covered under the existing application or account for any remaining animals in the continuation application. In 